\title{
Van der Waals and Casimir interactions between two graphene sheets
}

\author{
G. L. Klimchitskaya ${ }^{1}$ and V. M. Mostepanenko ${ }^{1}$ \\ ${ }^{1}$ Central Astronomical Observatory at Pulkovo of the \\ Russian Academy of Sciences, St.Petersburg, 196140, Russia
}

\begin{abstract}
The thermal free energy and pressure of dispersion interaction between two graphene sheets described by the Dirac model are calculated using the Lifshitz formula with reflection coefficients expressed via the polarization tensor. The obtained results for a pristine graphene are found to be in agreement with computations using Coulomb coupling between density fluctuations. For a graphene with nonzero mass gap parameter a qualitatively different behavior for the free energy and pressure is obtained. The Lifshitz formula with reflection coefficients expressed via the polarization tensor is used as a test for different computational approaches proposed in the literature for modeling the response function and conductivity of graphene at both zero and nonzero temperature.
\end{abstract}

PACS numbers: 78.67.Wj, 42.50.Lc, 65.80.Ck, 12.20.-m 


\section{INTRODUCTION}

The van der Waals and Casimir interactions, which are known under the generic name of dispersion forces, $\stackrel{1}{=}$ are caused by the vacuum and thermal fluctuations of the electromagnetic field. At shortest separations of a few nanometers dispersion forces are usually referred to as the van der Waals forces. At larger separations, when the relativistic retardation becomes important, it is customary to speak about the Casimir forces. In the last few years the fluctuation induced forces attracted much attention in the literature ${ }^{2-4}$ due to their prospective applications in both fundamental physics and nanotechnology. Specifically, a lot of experiments has been performed ${ }^{5-7}$ on measuring dispersion forces between metallic, dielectric and semiconductor surfaces spaced at separations from a few tens to a few hundreds nanometers.

Recently, special attention has been directed to carbon nanostructures, such as one-atomthick graphene sheets, carbon nanotubes, fullerenes etc. which possess unique mechanical, electrical and optical properties. $\stackrel{8}{9}$ These properties appear to be particularly promising to provide the basis for future carbon-based nanoelectronics. Keeping in mind that elements of nano- and microelectromechanical devices are separated by distances of the order of tens or hundreds nanometers, the dispersion forces acting between them are gaining in importance. For this reason, a lot of papers has been devoted to calculations of the van der Waals and Casimir forces between two carbon nanostructures and between a carbon nanostructure and a regular material body. $\underline{10}^{23}$ Particular attention has been given also the the Casimir-Polder interaction of different atoms and molecules with carbon nanostructures. $\underline{24}-26$

The many and varied formalisms were applied to calculate the van der Waals and Casimir forces between two graphene sheets. Here we center our attention on the approaches consistent with the most realistic Dirac model which assumes the linear dispersion relation of the graphene bands at low energy ${ }^{\underline{\underline{9}}}$ (there is also the so-called hydrodynamic model of graphene $13,14,27$ which does not take this property into account). Specifically, we compare the computational results obtained using the density fluctuation approach and the ran-

dom phase approximation, $10,16,20,21$ and using the conductivity of graphene modeled as a combination of Lorentz-type oscillators without account $\frac{18}{18}$ and with account 22 of spatial dispersion. It should be emphasized that the most straightforward formalism for calculation of the van der Waals and Casimir forces between graphene and different substances within 
the Dirac model is based on the Lifshitz theory and exploits the reflection coefficients of the electromagnetic oscillations on graphene expressed in terms of the polarization tensor in (2+1)-dimensional space-time. $\frac{15}{17}$ Using this formalism, the van der Waals and Casimir interactions between a graphene sheet and an ideal metal plane, $, \underline{15}, 17$ and between a graphene sheet and different atoms $\frac{26}{}$ and plates made of various real materials ${ }^{23}$ were computed. The case of graphene-graphene interaction remained, however, unexplored within this calculation approach.

The present paper is devoted to calculation of the graphene-graphene thermal van der Waals and Casimir interactions using the Lifshitz theory and the Dirac model for electronic properties of graphene. The reflection coefficients of the electromagnetic oscillations on graphene are expressed directly through the polarization tensor without recourse to the concept of dielectric permittivity. All calculations are performed for both gapless pristine graphene and graphene sheets characterized by some nonzero mass gap parameter. Note that the Dirac-type excitations in graphene become massive under the influence of electron-electron interaction, substrates, defects of structure and some other effects $\underline{\underline{9}} \underline{\underline{28}} \underline{\underline{31}}$ We calculate both the van der Waals and Casimir free energy per unit area and pressure as functions of separation between the graphene sheets and the van der Waals and Casimir pressure as a function of temperature. For a gapless graphene our results are in agreement with computations using Coulomb coupling between density fluctuations with subsequent thermal average. $\underline{\underline{16}}$ The latter approach is in fact equivalent $\underline{\underline{16}}$ to the nonretarded limit of the Lifshitz formula with the polarization of an isolated graphene sheet described in the random phase approximation. It was also shown, $\underline{\underline{16}}$ that retardation effects are of only minor importance for graphene. For graphene sheets with nonzero mass gap parameter we obtain a qualitatively different behavior for the free energy and pressure, as compared to the pristine graphene. In this case the character of force depends on the relationship between the mass gap parameter and the temperature. We provide a discussion concerning the comparison of our results with other results obtained in the literature for graphene-graphene van der Waals and Casimir interactions using the Dirac model $\underline{10}, \underline{18,20} \underline{22}$

The paper is organized as follows. In Sec. II we begin with the Lifshitz formula containing the reflection coefficients derived using the Dirac model. Then we present our results for the free energy of graphene-graphene van der Waals and Casimir interactions per unit area and pressure as functions of separation and temperature. Section III contains the comparison of 
our results with the results by others obtained at both zero and nonzero temperature. In Sec. IV the reader will find our conclusions and discussion. For simplicity in comparisons with the classical limit, we preserve the fundamental constants (the Planck constant $\hbar$, the velocity of light $c$ and the Boltzmann constant $k_{B}$ ) in all mathematical expressions.

\section{DISPERSION INTERACTION OF TWO GRAPHENE SHEETS DESCRIBED BY THE DIRAC MODEL}

The free energy of the van der Waals and Casimir interactions per unit area of two parallel graphene sheets separated by a distance $a$ at thermal equilibrium with an environment at temperature $T$ is given by the Lifshitz formula ${ }^{1-\underline{\underline{7}}}$

$$
\mathcal{F}(a, T)=\frac{k_{B} T}{8 \pi a^{2}} \sum_{l=0}^{\infty} \int_{\zeta_{l}}^{\infty} y d y\left\{\ln \left[1-r_{\mathrm{TM}}^{2}\left(i \zeta_{l}, y\right) e^{-y}\right]+\ln \left[1-r_{\mathrm{TE}}^{2}\left(i \zeta_{l}, y\right) e^{-y}\right]\right\} .
$$

Here, $\zeta_{l}$ are the dimensionless Matsubara frequencies connected with the dimensional ones $\xi_{l}=2 \pi k_{B} T l / \hbar$ (where $l=0,1,2, \ldots$ ) by the equality $\zeta_{l}=\xi_{l} / \omega_{c}$ with $\omega_{c}=c /(2 a)$. The dimensionless variable $y$ is connected with the magnitude of the projection of the wave vector on the plane of graphene, $k_{\perp}$, by the equality $y=2 a\left(k_{\perp}^{2}+\xi_{l}^{2} / c^{2}\right)^{1 / 2}$. The prime near the summation sign means that the term with $l=0$ should be taken with a factor $1 / 2$.

In the framework of the Dirac model at $T \neq 0$ the reflection coefficients on graphene for two independent polarizations of the electromagnetic field, transverse magnetic (TM) and transverse electric (TE), were found in Ref. 17 . In terms of our dimensionless variables they are given by 23,26

$$
\begin{aligned}
& r_{\mathrm{TM}}\left(i \zeta_{l}, y\right)=\frac{y \tilde{\Pi}_{00}}{y \tilde{\Pi}_{00}+2\left(y^{2}-\zeta_{l}^{2}\right)}, \\
& r_{\mathrm{TE}}\left(i \zeta_{l}, y\right)=-\frac{\left(y^{2}-\zeta_{l}^{2}\right) \tilde{\Pi}_{t r}-y^{2} \tilde{\Pi}_{00}}{\left(y^{2}-\zeta_{l}^{2}\right)\left(\tilde{\Pi}_{t r}+2 y\right)-y^{2} \tilde{\Pi}_{00}}
\end{aligned}
$$

where the dimensionless components of the polarization tensor in $(2+1)$-dimensional space-

time are expressed as $\tilde{\Pi}_{00, t r}=2 a \prod_{00, t r} / \hbar$ through the dimensional ones and trace stands for the sum of spatial components $\Pi_{1}^{1}$ and $\Pi_{2}^{2}$.

The explicit expression for the 00-component of the polarization tensor for graphene with a nonzero mass gap parameter $\Delta$ but zero chemical potential can be written in the 
form $17,23,26$

$$
\begin{aligned}
& \tilde{\Pi}_{00}\left(i \zeta_{l}, y\right)=8 \alpha\left(y^{2}-\zeta_{l}^{2}\right) \int_{0}^{1} d x \frac{x(1-x)}{\left[\tilde{\Delta}^{2}+x(1-x) f\left(\zeta_{l}, y\right)\right]^{1 / 2}}+\frac{8 \alpha}{\tilde{v}_{F}^{2}} \int_{0}^{1} d x \\
& \quad \times\left\{\frac{\tau}{2 \pi} \ln \left[1+2 \cos (2 \pi l x) e^{-g\left(\tau, \zeta_{l}, y\right)}+e^{-2 g\left(\tau, \zeta_{l}, y\right)}\right]-\frac{\zeta_{l}}{2}(1-2 x) \frac{\sin (2 \pi l x)}{\cosh g\left(\tau, \zeta_{l}, y\right)+\cos (2 \pi l x)}\right. \\
& \left.+\frac{\tilde{\Delta}^{2}+\zeta_{l}^{2} x(1-x)}{\left[\tilde{\Delta}^{2}+x(1-x) f\left(\zeta_{l}, y\right)\right]^{1 / 2}} \frac{\cos (2 \pi l x)+e^{-g\left(\tau, \zeta_{l}, y\right)}}{\cosh g\left(\tau, \zeta_{l}, y\right)+\cos (2 \pi l x)}\right\} .
\end{aligned}
$$

Here, $\alpha=e^{2} /(\hbar c)$ in the fine-structure constant, $\tilde{\Delta}=\Delta /\left(\hbar \omega_{c}\right)$ is the dimensionless mass gap parameter, $\tilde{v}_{F}=v_{F} / c \approx 1 / 300$ is the dimensionless Fermi velocity, and $\tau=2 \pi T / T_{\text {eff }}=$ $4 \pi a k_{B} T /(\hbar c)$, where $T_{\text {eff }}$ is the so-called effective temperature. The dimensionless functions $f$ anf $g$ contained in Eq. (3) are defined as

$$
\begin{aligned}
& f\left(\zeta_{l}, y\right)=\tilde{v}_{F}^{2} y^{2}+\left(1-\tilde{v}_{F}^{2}\right) \zeta_{l}^{2} \\
& g\left(\tau, \zeta_{l}, y\right)=\frac{2 \pi}{\tau}\left[\tilde{\Delta}^{2}+x(1-x) f\left(\zeta_{l}, y\right)\right]^{1 / 2}
\end{aligned}
$$

The explicit expression for the trace of the polarization tensor is given by $\underline{17}, 23,26$

$$
\begin{aligned}
& \tilde{\Pi}_{t r}\left(i \zeta_{l}, y\right)=8 \alpha\left[y^{2}+f\left(\zeta_{l}, y\right)\right] \int_{0}^{1} d x \frac{x(1-x)}{\left[\tilde{\Delta}^{2}+x(1-x) f\left(\zeta_{l}, y\right)\right]^{1 / 2}}+\frac{8 \alpha}{\tilde{v}_{F}^{2}} \int_{0}^{1} d x \\
& \quad \times\left\{\frac{\tau}{2 \pi} \ln \left[1+2 \cos (2 \pi l x) e^{-g\left(\tau, \zeta_{l}, y\right)}+e^{-2 g\left(\tau, \zeta_{l}, y\right)}\right]\right. \\
& -\frac{\zeta_{l}\left(1-2 \tilde{v}_{F}^{2}\right)}{2}(1-2 x) \frac{\sin (2 \pi l x)}{\cosh g\left(\tau, \zeta_{l}, y\right)+\cos (2 \pi l x)} \\
& \left.+\frac{\tilde{\Delta}^{2}+x(1-x)\left[\left(1-\tilde{v}_{F}^{2}\right)^{2} \zeta_{l}^{2}-\tilde{v}_{F}^{4} y^{2}\right]}{\left[\tilde{\Delta}^{2}+x(1-x) f\left(\zeta_{l}, y\right)\right]^{1 / 2}} \frac{\cos (2 \pi l x)+e^{-g\left(\tau, \zeta_{l}, y\right)}}{\cosh g\left(\tau, \zeta_{l}, y\right)+\cos (2 \pi l x)}\right\} .
\end{aligned}
$$

The Lifshitz formula for the pressure of dispersion interaction between two parallel graphene sheets takes the form

$$
P(a, T)=-\frac{k_{B} T}{8 \pi a^{3}} \sum_{l=0}^{\infty} \int_{\zeta_{l}}^{\infty} y^{2} d y\left\{\left[r_{\mathrm{TM}}^{-2}\left(i \zeta_{l}, y\right) e^{y}-1\right]^{-1}+\left[r_{\mathrm{TE}}^{-2}\left(i \zeta_{l}, y\right) e^{y}-1\right]^{-1}\right\} .
$$

We begin with computations of the free energy of dispersion interaction between two graphene sheets using Eqs. (1)-(5) . In Fig. 1(a,b) we plot the computational results for the 
free energy per unit area $\mathcal{F}$ at $T=300 \mathrm{~K}$ normalized to the Casimir energy between two ideal metal planes at zero temperature, $E_{C}(a)=-\pi^{2} \hbar c /\left(720 a^{3}\right)$, in the separation region (a) from 5 to $1000 \mathrm{~nm}$ and (b) on an enlarged scale from 5 to $100 \mathrm{~nm}$. The bottom and top solid lines in Fig. 1(a) show the ratio $\mathcal{F} / E_{C}$ at $T=300 \mathrm{~K}$ for graphene sheets with the mass gap parameter $\Delta=0.1 \mathrm{eV}$ and $0 \mathrm{eV}$, respectively. In Fig. 1(b) the quantity $\mathcal{F} / E_{C}$ at $T=300 \mathrm{~K}$ is shown by the solid lines from bottom to top for $\Delta=0.1 \mathrm{eV}, 0.05 \mathrm{eV}$ and $0 \mathrm{eV}$, respectively, and at $T=0 \mathrm{~K}$ by the bottom and top long-dashed lines for $\Delta=0.1 \mathrm{eV}$ and $0 \mathrm{eV}$, respectively. As can be seen in Fig. 11(a,b), at short separations the free energy of graphene-graphene dispersion interaction is much smaller than the Casimir interaction between two ideal metal planes, but becomes relatively large with increasing separation distance. The nonzero gap parameter depending on its value exerts some influence on the free energy. From Fig. 1(b) it is seen that already at short separations from 10 to $20 \mathrm{~nm}$ the computational results at $T=300 \mathrm{~K}$ differ considerably from those at $T=0 \mathrm{~K}$. This means that for graphene thermal effects should be taken into account not only for the Casimir force, but for the nonrelativistic van der Waals force as well. 16

The computations of the van der Waals and Casimir pressures between two graphene sheets were performed using Eqs. (2) -(6) . In Fig. 2(a,b) the computational results for the pressure $P$ at $T=300 \mathrm{~K}$ normalized to the Casimir pressure between two ideal metal planes at zero temperature, $P_{C}(a)=-\pi^{2} \hbar c /\left(240 a^{4}\right)$, are plotted in the separation region (a) from 5 to $1000 \mathrm{~nm}$ and (b) on an enlarged scale from 5 to $100 \mathrm{~nm}$. The meaning of the solid and the long-dashed lines is the same as in Fig. 11(a,b). From Fig. 2(a,b) it can be concluded that the pressure of graphene-graphene dispersion interaction possesses all the same properties as discussed above in the case of free energy. Specifically, at $T=300 \mathrm{~K}$ the thermal effect becomes large enough at short separations of about 10-20 nm and its role quickly increases with increasing separation depending on the value of $\Delta$.

The top solid line in Fig. 2(b) related to the case of pristine graphene $(\Delta=0)$ within the separation region from 5 to $100 \mathrm{~nm}$ is in agreement with the solid line in Fig. 2 of Ref. $\underline{16}$ obtained using Coulomb couplings between density fluctuations with subsequent thermal average. As was shown, $\underline{16}$ thermal effects in the pressure of dispersion interaction for graphene are noticeable already in the van der Waals regime, i.e., at distances of tens of nanometers at room temperature $T=300 \mathrm{~K}$. From the comparison of the top solid and top long-dashed 
lines in Fig. 2(b) one can calculate the relative thermal correction to the Casimir pressure

$$
\delta_{T} P(a)=\frac{P(a, T)-P_{C}(a)}{P_{C}(a)}
$$

at different separations for a gapless graphene. Thus, at $a=10,20,50$, and $100 \mathrm{~nm}$ one obtains $\delta_{T} P(a)=5.6 \%, 20.8 \%, 89.4 \%$, and $228 \%$, respectively [remind that for customary metallic and dielectric materials thermal correction becomes large only at separations of a few micrometers comparable with the so-called thermal length $\left.\hbar^{4} \hbar c /\left(2 k_{B} T\right)\right]$.

Our results show that for graphene sheets with a nonzero mass gap parameter the thermal effect (with exception of only shortest separations) becomes even larger. Thus, from the comparison of the bottom solid line with the bottom long-dashed line in Fig. 2(b) (graphene sheets with $\Delta=0.1 \mathrm{eV}$ ) at separations $a=10,20,50$, and $100 \mathrm{~nm}$ we obtain $\delta_{T} P(a)=$ $4.7 \%, 25.3 \%, 200 \%$, and $893 \%$. This makes feasible an observation of the thermal effect in the dispersion interaction of two graphene sheets and even an estimation of the mass gap parameter by the results of force measurements.

The role of the mass gap parameter in the temperature dependence of the van der Waals and Casimir pressure between two graphene sheets is illustrated in Fig. 3. Here, the separation distance is fixed at $a=30 \mathrm{~nm}$ and the pressure magnitudes are plotted as functions of temperature for $\Delta=0.1 \mathrm{eV}, 0.05 \mathrm{eV}, 0.01 \mathrm{eV}$, and $0 \mathrm{eV}$ from bottom to top, respectively. As can be seen in Fig. 3, for any nonzero mass gap parameter there is some temperature region where the pressure magnitude remains nearly constant when the temperature increases. The larger is the mass gap parameter, the wider is this temperature region. Thus, for $\Delta=0.1 \mathrm{eV}$, $0.05 \mathrm{eV}$, and $0.01 \mathrm{eV}$ the pressure magnitude remains nearly constant (less than $1 \%$ increase) up to $T=155 \mathrm{~K}, 95 \mathrm{~K}$, and $45 \mathrm{~K}$, respectively. Note that the same characteristic dependence on $\Delta$ for dispersion interactions of graphene with atomic systems and material plates made of dielectrics and metals was found earlier. ${ }^{23,26}$ The Casimir free energy remains nearly constant if the condition $k_{B} T \ll \Delta$ is satisfied with a large safety margins. Under the condition $\Delta \lesssim k_{B} T$ the thermal correction becomes relatively large.

The results of numerical computations presented above can be supplemented by the asymptotic behaviors of the free energy and pressure at large and short separations (high and low temperatures). In the case of large separations (high temperatures) the asymptotic behavior of the Casimir free energy and pressure is determined by the zero-frequency contribution to the Lifshitz formulas (1) and (6). Using the asymptotic expressions for the 
reflection coefficients obtained earlier, $, 17,23$ one arrives at the following Casimir free energy per unit area in the large separation (high temperature) limit

$$
\mathcal{F}(a, T)=-\frac{k_{B} T \zeta(3)}{16 \pi a^{2}}\left[1-\frac{\tilde{v}_{F}^{2} \hbar c}{4 \alpha a k_{B} T \ln \left(2 \cosh \frac{\Delta}{2 k_{B} T}\right)}\right],
$$

where $\zeta(z)$ is the Riemann zeta function. Note that the second term in the square brackets of Eq. (8) is small comparing with unity due to the smallness of $\tilde{v}_{F}$. In a similar way for the Casimir pressure at large separations (high temperatures) it holds

$$
P(a, T)=-\frac{k_{B} T \zeta(3)}{8 \pi a^{3}}\left[1-\frac{3 \tilde{v}_{F}^{2} \hbar c}{8 \alpha a k_{B} T \ln \left(2 \cosh \frac{\Delta}{2 k_{B} T}\right)}\right] .
$$

The asymptotic expressions (81) and (9) are in a good agreement with the results of numerical computations shown in Figs. 1(a) and 2(a). As an example, for $\Delta=0, T=300 \mathrm{~K}$ the pressure values calculated using Eq. (9) agree with computations in the limits of $1 \%$ at separations $a>370 \mathrm{~nm}$. In the limits of $5 \%$ the analytic and computational results agree at $a>150 \mathrm{~nm}$. For graphene with nonzero mass gap parameter the asymptotic expressions (8) and (9) become applicable starting from larger separation distances. Thus, for $\Delta=0.1 \mathrm{eV}$, $T=300 \mathrm{~K}$ the agreement between analytic and numerical computations in the limits of $5 \%$ is achieved at $a \geq 800 \mathrm{~nm}$. Note that the first term on the right-hand side of Eq. (9) was obtained in Ref. 16 for a gapless graphene with $\Delta=0$. The second terms in Eqs. (8) and (9) provide first corrections to the previously obtained result and generalize it to the case of graphene with a nonzero mass gap parameter. It should be stressed that the main (first) terms on the right-hand side of Eqs. (8) and (9) correspond to the so-called classical limit ${ }^{32}$ because they do not depend on the Planck constant.

The case of two graphene sheets interacting via the nonthermal van der Waals interaction is restricted to the shortest separations from 1 to $3 \mathrm{~nm}$. Here, using Eqs. (11) and (6) at $T=0$, $\Delta=0$, one obtains

$$
E(a)=\mathcal{F}(a, 0)=-\frac{C}{a^{3}}, \quad P(a, 0)=-\frac{3 C}{a^{4}},
$$


where the constant $C$ is given by

$$
\begin{gathered}
C=\frac{\hbar c}{32 \pi^{2}} \int_{0}^{\infty} y d y \int_{0}^{y} d \zeta\left\{\ln \left[1-\left(\frac{\alpha \pi y}{2 \sqrt{f(\zeta, y)}+\alpha \pi y}\right)^{2} e^{-y}\right]\right. \\
\left.+\ln \left[1-\left(\frac{\alpha \pi \sqrt{f(\zeta, y)}}{2 y+\alpha \pi \sqrt{f(\zeta, y)}}\right)^{2} e^{-y}\right]\right\}
\end{gathered}
$$

Numerical computations of the integrals in Eq. (11) lead to

$$
C=0.02101 \frac{\hbar c}{32 \pi^{2}}=2.103 \times 10^{-30} \mathrm{~J} \mathrm{~m}=0.131 \mathrm{eV} \AA .
$$

The result (12) is in agreement with earlier obtained ${ }^{16}$ estimation $3 C \sim 0.4 \mathrm{eV} \AA$ for the van der Waals pressure in Eq. (10). In the next section the above results are compared with other results obtained in the literature using the Dirac model.

\section{COMPARISON OF DIFFERENT RESULTS FOR DISPERSION INTERAC- TION BETWEEN GRAPHENE SHEETS}

We begin with the van der Waals interaction between two graphene sheets at zero temperature. In the first paper devoted to this subject ${ }^{10}$ the van der Waals energy per unit area, as in Eq. (10), was obtained with the coefficient $C$ equal to $C=0.288 \mathrm{eV} \AA$. This is more than twice as large as our value in Eq. (12).

In a later paper ${ }^{20}$ a smaller value for this coefficient was obtained $C=2.156 \times 10^{-30} \mathrm{~J} \mathrm{~m}=$ $0.134 \mathrm{eV} \AA$ in a rather good agreement with our result (12). A slightly larger value computed $^{20}$ might be explained by slightly different value of the used Fermi velocity $\left(v_{F}=8.73723 \times 10^{5} \mathrm{~m} / \mathrm{s}\right.$ instead of $v_{F}=10^{6} \mathrm{~m} / \mathrm{s}$ as in our work). Although the formalism used 20 is nonretarded, a good agreement with our fully relativistic computations was achieved. This again confirms the conclusion 16 that relativistic retardation does not play a major role for graphene.

Now we discuss the computational results obtained at zero temperature by using the models for conductivity of graphene in terms of Lorentz-type oscillators. 18 By assuming that over a relatively wide range of photon frequencies up to $3 \mathrm{eV}$ the graphene conductivity is approximately constant equal to $\sigma_{0}=e^{2} /(4 \hbar)$, it was found $\frac{18}{4}$ that the Casimir pressure is given by the second equality in Eq. (10). For the constant in this equality it was derived $\underline{18}$ 
$3 C=6.88 \times 10^{-30} \mathrm{~J} \mathrm{~m}=0.430 \mathrm{eV} \AA$ leading to the following constant for the Casimir energy per unit area $C=2.29 \times 10^{-30} \mathrm{~J} \mathrm{~m}=0.143 \mathrm{eV} \AA$. This is a slightly larger value than was obtained in Eq. (12) on the basis of the Dirac model.

The conductivity of graphene over a wider range of frequencies was also modeled 18 by assuming that its optical properties are very similar to the in-plane optical properties of graphite. The latter have been mapped to a series of Lorentz oscillators with the Drude term over the frequency range from 0.1 to $40 \mathrm{eV}$. As a result, the Casimir pressure between two graphene sheets was computed using the Lifshitz formula. The computational results normalized to the Casimir pressure between two ideal metal planes taken from Fig. 4(b) in Ref. $\frac{18}{}$ are plotted in our Fig. 4 by the gray short-dashed line. In the same figure, the gray solid line shows the results obtained $\frac{18}{18}$ under an assumption of constant graphene conductivity $\sigma_{0}\left(P / P_{C}=0.00529\right)$. As can be seen in Fig. 4, an assumption of the frequency-dependent conductivity of graphene leads to significant deviations at short separation distances. For the sake of convenience in Fig. 4 we also present the discussed above results of Ref. 20 (the dotted line which corresponds to $\left.P / P_{C}=0.00497\right)$ and our results for the gapless graphene sheets and for graphene with the mass gap parameter $\Delta=0.1 \mathrm{eV}$ (they are shown by the top and bottom long-dashed lines, respectively). For a gapless graphene it holds $P / P_{C}=0.00485$.

It should be emphasized that although the formalism of reflection coefficients expressed in terms of the polarization tensor provides a reliable test for any alternative approach, at room temperature the application region of the results computed at zero temperature is restricted to only the shortest separations below a few nanometers due to large thermal effects discussed in Sec. II. Because of this, below we compare our results with those computed in the literature taking nonzero temperature into account.

Using the Lifshitz theory and some version of the response function of graphene in the random phase approximation, the Casimir free energy between two graphene sheets was computed $^{21}$ within a wide range of separations at room temperature $T=300 \mathrm{~K}$. We show the computational results taken from Fig. 4 of Ref. 21 in our Fig. 5(a) by the dashed line for the pristine graphene. For comparison purposes the solid line in Fig. 5)(a) reproduces our results for a pristine graphene already shown by the top solid line in Fig. 1(a) in another form. As can be seen in Fig. 5(a), the dashed line deviates significantly from the solid line obtained using the Dirac model and the Lifshitz theory with the reflection coefficients expressed via the polarization tensor (remind that the results shown by the solid line are 
in agreement with those obtained in Ref. $\left.{ }^{16}\right)$. At asymptotically large separations the free energy of the Casimir interaction is given by the zero-frequency term of the Lifshitz formula (11) and for two pristine graphene sheets the formalism using the temperature-independent response function of graphene leads to $\mathcal{F} \sim 1 / a^{4}$ (see Table I in Ref. ${ }^{21}$ ), whereas Eq. (8) demonstrates the classical limit, $\mathcal{F} \sim-k_{B} T / a^{2}$, as it should be at large separations (high temperatures). According to Ref. ${ }^{21}$ the reason for deviation from the classical limit is that the used formalism ${ }^{21}$ takes into account the direct temperature effects, as given by the finitetemperature Lifshitz formula, but neglects the indirect temperature effects arising from the temperature dependence of the dielectric response of graphene (the latter are taken into account by the polarization tensor at nonzero temperature). Furthermore, Ref. 21 expects that for graphene with high doping concentration the indirect temperature effects at room temperature should be negligibly small. Thus, for graphene with doping electron density $10^{16} \mathrm{~m}^{-2}$, using the formalism accounting for only the direct temperature effects it was found ${ }^{21}$ that $\mathcal{F} \sim 1 / a^{2}$ at separations $a>1 \mu \mathrm{m}$ in accordance to the classical limit. From this it was concluded that for undoped graphene sheets the formalism with neglected temperature dependence of the dielectric response of graphene is applicable only at vanishingly small separations of about a few angströms. ${ }^{21}$ These suppositions of Ref. ${ }^{21}$ concerning the origin of disagreement between their results and the respective results of Ref. 16 , coinciding with our results, invite further investigation.

Now we consider computations of the thermal Casimir pressure between two graphene sheets with both zero and nonzero mass gap parameter ${ }^{22}$ In this approach the dielectric properties of graphene were described via the optical conductivity calculated using the Kubo formalism. The computationsl results ${ }^{22}$ for the van der Waals and Casimir pressure normalized to the Casimir pressure between two ideal metal planes are shown by the bottom and top dashed lines in Fig. 5(b) for graphene sheets with $\Delta=0.1 \mathrm{eV}$ and $\Delta=0 \mathrm{eV}$, respectively. These results are recalculated from Fig. 2(a) in Ref. $\frac{22}{\text { by }}$ using their Eq. (10). For comparison purposes our results for the normalized van der Waals and Casimir pressure are reproduced by the three solid lines from bottom to top for $\Delta=0.1 \mathrm{eV}, 0.05 \mathrm{eV}$, and $0 \mathrm{eV}$, respectively. As discussed in Sec. II, the top solid line is in agreement with respective computational results of Ref..$^{16}$ where the case of gapless graphene was also considered using an alternative formalism. From Fig. [5) (b) it is seen that the dashed lines deviate significantly from the respective solid lines especially at short separations below a few tens of nanometers. 
In the limiting case of large separation distances (high temperatures) the used formalism leads to one half of the result for two ideal metal planes, i.e., the obtained Casimir pressure coincides with the first term of our asymptotic expression (91). Thus, this formalism, although not enough precise at moderate and short separations, satisfies the classical limit.

\section{CONCLUSIONS AND DISCUSSION}

In the foregoing we have investigated the van der Waals and Casimir free energy and pressure between two graphene sheets interacting via the zero-point and thermal fluctuations of the electromagnetic field using the Dirac model of graphene. This was done with the help of the Lifshitz theory where the reflection coefficients were expressed via the components of the polarization tensor in $(2+1)$-dimensional space-time. In so doing both the pristine graphene and the gapped graphene were considered.

It is common knowledge that graphene and graphene-based nanostructures are the materials of high promise for many prospective applications in micro- and nanoelectronics and, more widely, in nanotechnology. Because of this, it is of high priority to have reliable theoretical predictions for the van der Waals and Casimir interactions between graphene sheets and other carbon nanostructures spaced at separations below a micrometer. At the present time, suspended graphene membranes of sufficiently large area are already available.$^{33}$ It is highly probable that measurements of dispersion interaction between graphene sheets and other carbon-based nanostructures will be performed in the immediate future. In this situation the reliable and confirmed theoretical results for a simplest system, such as two graphene sheets, are urgently needed.

The Lifshitz theory with reflection coefficients expressed in terms of the polarization tensor provides a straightforward formalism for the comparison with other approaches. We have calculated the free energy and pressure of dispersion interaction between two graphene sheets with zero mass gap parameter and arrived to the results in agreement with obtained earlier ${ }^{16}$ using Coulomb coupling between density fluctuations. Specifically, the existence of large thermal effect for two graphene sheets was confirmed as well as the asymptotic behaviors of the free energy and pressure at short and large separations. We have also generalized these results to the case of graphene with a nonzero mass gap parameter. In this

case the thermal van der Waals and Casimir interactions between two graphene sheets are 
shown to depend on an interrelation between the temperature and the mass gap parameter. The results obtained were compared with some other results in the literature for graphenegraphene interaction at both zero and nonzero temperature. This allowed to clarify the regions of applicability of several approaches to the definition of response function and conductivity of graphene starting from the measure of agreement between these approaches and the Lifshitz theory with reflection coefficients found using the Dirac model.

In the future it would be topical to investigate the van der Waals and Casimir interactions between graphene sheets deposited on material substrates. This subject is of much interest for experiments in preparation. It is of interest also to take into account deviations of the dispersion relation for graphene quasiparticles from linearity at high energy.

\section{Acknowledgments}

The authors are grateful to M. Bordag for stimulating discussions.

1 J. Mahanty and B. W. Ninham, Dispersion Forces (Academic Press, London, 1976).

2 M. Kardar and R. Golestanian, Rev. Mod. Phys. 71, 1233 (1999).

3 V. A. Parsegian, Van der Waals Forces: A Handbook for Biologists, Chemists, Engineers, and Physicists (Cambridge University Press, Cambridge, 2005).

4 M. Bordag, G. L. Klimchitskaya, U. Mohideen, and V. M. Mostepanenko, Advances in the Casimir Effect (Oxford University Press, Oxford, 2009).

5 G. L. Klimchitskaya, U. Mohideen, and V. M. Mostepanenko, Rev. Mod. Phys. 81, 1827 (2009).

6 A. W. Rodriguez, F. Capasso, and S. G. Johnson, Nature Photon. 5, 211 (2011).

7 G. L. Klimchitskaya, U. Mohideen, and V. M. Mostepanenko, Int. J. Mod. Phys. B 25, 171 (2011).

8 M. S. Dresselhaus, Physica Status Solidi (b) 248, 1566 (2011).

9 A. H. Castro Neto, F. Guinea, N. M. R. Peres, K. S. Novoselov, and A. K. Geim, Rev. Mod. Phys. 81, 109 (2009).

10 J. F. Dobson, A. White, and A. Rubio, Phys. Rev. Lett. 96, 073201 (2006).

11 I. V. Bondarev and Ph. Lambin, Phys. Rev. B 70, 035407 (2004). 
12 E. V. Blagov, G. L. Klimchitskaya, and V. M. Mostepanenko, Phys. Rev. B 71, 235401 (2005).

13 M. Bordag, J. Phys. A: Math. Gen. 39, 6173 (2006).

14 M. Bordag, B. Geyer, G. L. Klimchitskaya, and V. M. Mostepanenko, Phys. Rev. B 74, 205431 (2006).

15 M. Bordag, I. V. Fialkovsky, D. M. Gitman, and D. V. Vassilevich, Phys. Rev. B 80, 245406 (2009).

16 G. Gómez-Santos, Phys. Rev. B 80, 245424 (2009).

17 I. V. Fialkovsky, V. N. Marachevsky, and D. V. Vassilevich, Phys. Rev. B 84, 035446 (2011).

18 D. Drosdoff and L. M. Woods, Phys. Rev. B 82, 155459 (2010).

19 D. Drosdoff and L. M. Woods, Phys. Rev. A 84, 062501 (2011).

20 B. E. Sernelius, Europhys. Lett. 95, 57003 (2011).

21 J. Sarabadani, A. Naji, R. Asgari, and R. Podgornik, Phys. Rev. B 84, 155407 (2011).

22 D. Drosdoff, A. D. Phan, L. M. Woods, I. V. Bondarev, and J. F. Dobson, Eur. Phys. J. B 85, $365(2012)$.

23 M. Bordag, G. L. Klimchitskaya, and V. M. Mostepanenko, Phys. Rev. B 86, 165429 (2012).

24 Yu. V. Churkin, A. B. Fedortsov, G. L. Klimchitskaya, and V. A. Yurova, Phys. Rev. B 82, $165433(2010)$.

25 T. E. Judd, R. G. Scott, A. M. Martin, B. Kaczmarek, and T. M. Fromhold, New. J. Phys. 13, $083020(2011)$.

26 M. Chaichian, G. L. Klimchitskaya, V. M. Mostepanenko, and A. Tureanu, Phys. Rev. A 86, 012515 (2012).

27 G. Barton, J. Phys. A 38, 2997 (2005).

28 S. A. Jafari, J. Phys.: Cond. Mat. 24, 205802 (2012).

29 P. K. Pyatkovskiy, J. Phys.: Cond. Mat. 21, 025506 (2009).

30 V. P. Gusynin, S. G. Sharapov, and J. P. Carbotte, New J. Phys. 11, 095013 (2009).

31 V. P. Gusynin and S. G. Sharapov, Phys. Rev. B 73, 245411 (2006).

32 J. Feinberg, A. Mann, and M. Revzen, Ann. Phys. (N.Y.) 288, 103 (2001).

33 B. Alemán, W. Regan, S. Aloni, V. Altoe, N. Alem, C. Girit, B. Geng, L. Maserati, M. Crommie, F. Wang, and A. Zettl, ACS Nano 4, 4762 (2010). 

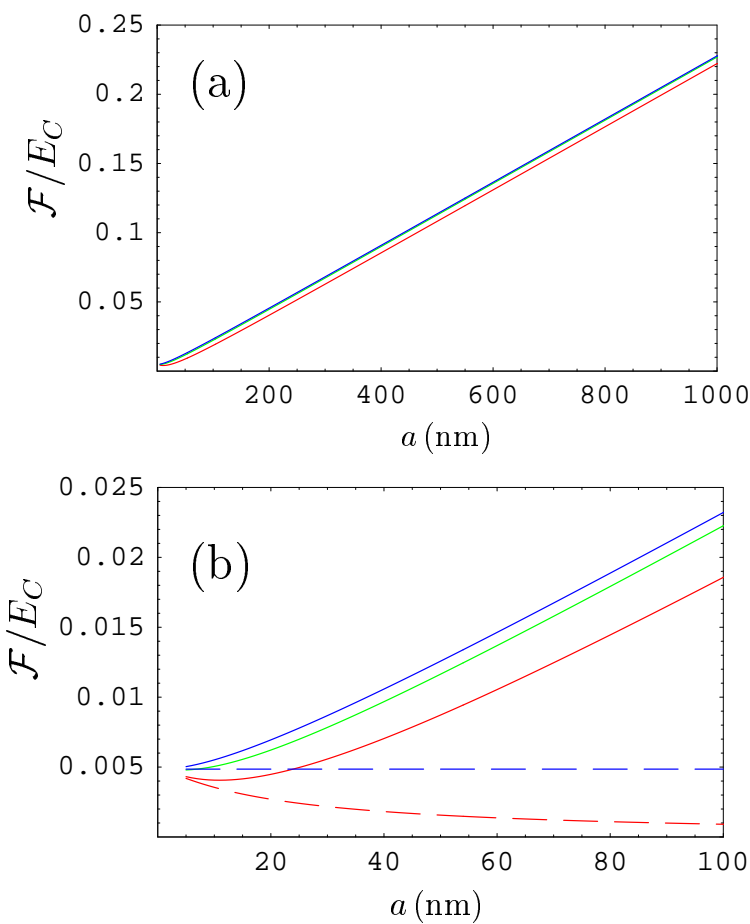

FIG. 1: (Color online) The normalized van der Waals and Casimir free energy of graphene-graphene interaction per unit area as a function of separation (a) from 5 to $1000 \mathrm{~nm}$ and (b) from 5 to $100 \mathrm{~nm}$. The solid lines from bottom to top are for $T=300 \mathrm{~K}$ and the mass gap parameter $\Delta=0.1 \mathrm{eV}$, $0.05 \mathrm{eV}$, and $0 \mathrm{eV}$, respectively. The bottom and top long-dashed lines are plotted at $T=0$ for $\Delta=0.1 \mathrm{eV}$ and $0 \mathrm{eV}$, respectively. 

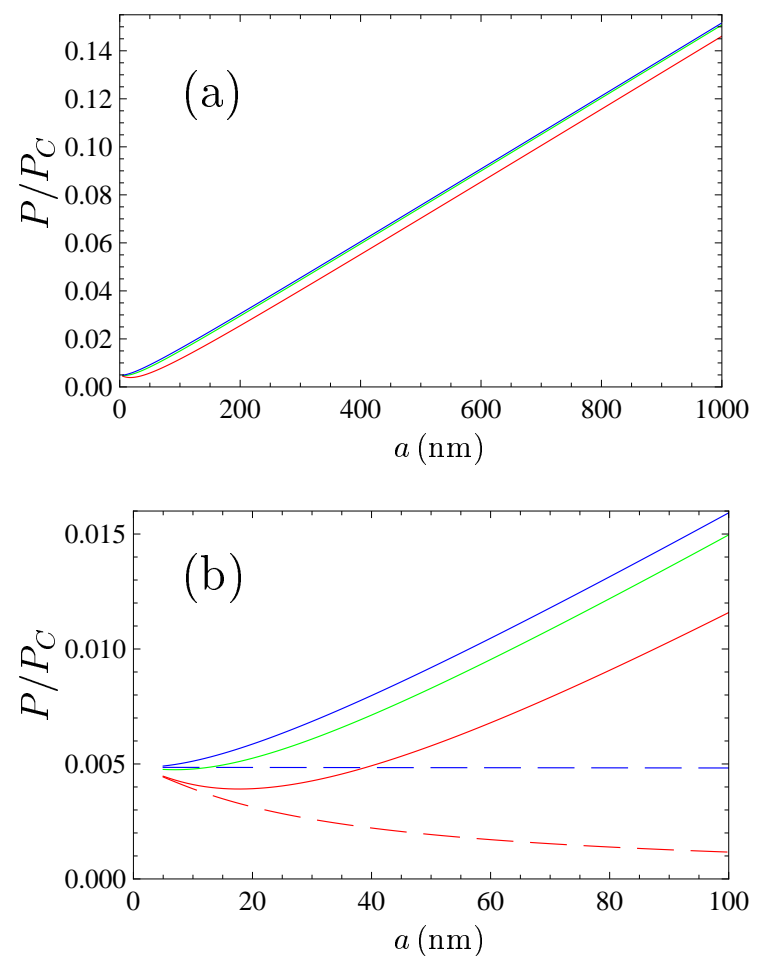

FIG. 2: (Color online) The normalized van der Waals and Casimir pressure of graphene-graphene interaction as a function of separation (a) from 5 to $1000 \mathrm{~nm}$ and (b) from 5 to $100 \mathrm{~nm}$. The solid lines from bottom to top are for $T=300 \mathrm{~K}$ and the mass gap parameter $\Delta=0.1 \mathrm{eV}, 0.05 \mathrm{eV}$, and $0 \mathrm{eV}$, respectively. The bottom and top long-dashed lines are plotted at $T=0$ for $\Delta=0.1 \mathrm{eV}$ and $0 \mathrm{eV}$, respectively. 


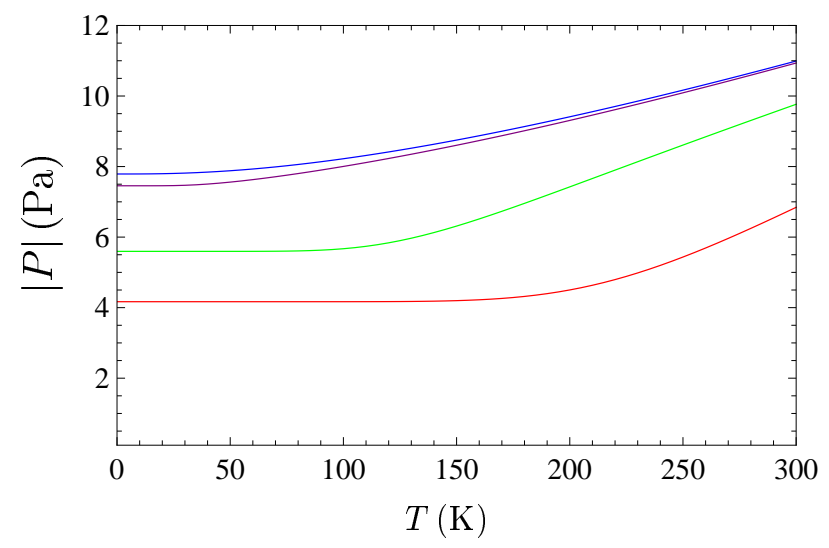

FIG. 3: (Color online) The van der Waals and Casimir pressure for graphene-graphene interaction at $a=30 \mathrm{~nm}$ as a function of temperature. The lines from bottom to top are for the mass gap parameter $\Delta=0.1 \mathrm{eV}, 0.05 \mathrm{eV}, 0.01 \mathrm{eV}$, and $0 \mathrm{eV}$, respectively.

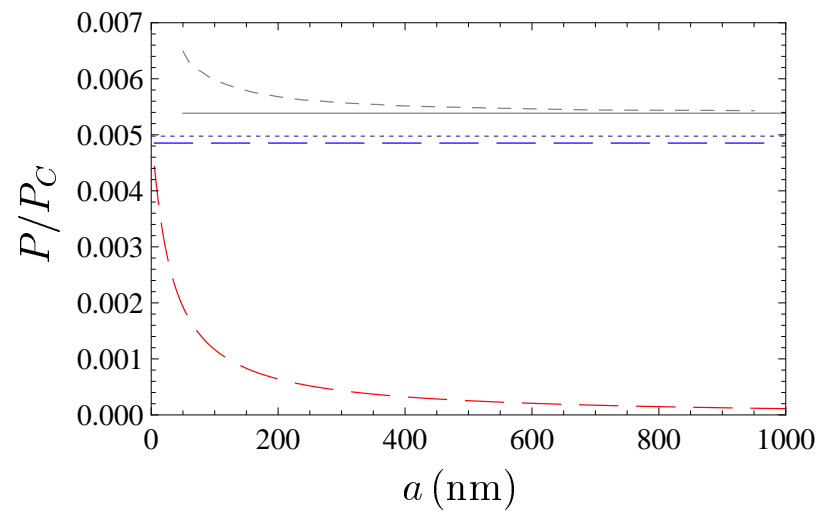

FIG. 4: (Color online) The normalized van der Waals and Casimir pressure of graphene-graphene interaction at $T=0$ as a function of separation. The long-dashed lines show our results for $\Delta=$ $0.1 \mathrm{eV}$ (bottom) and $0 \mathrm{eV}$ (top). The dotted line shows the results of Ref. $\underline{20}$. The solid and dashed gray lines represent the results of Ref 18 for a constant and frequency-dependent conductivity of graphene, respectively. 

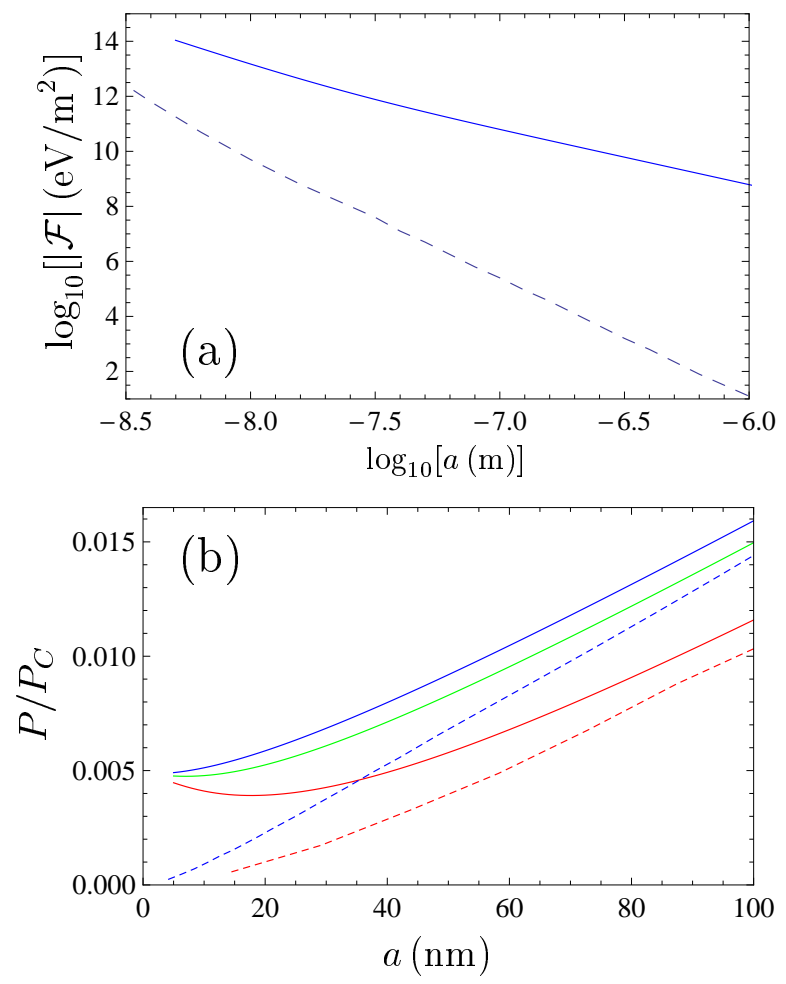

FIG. 5: (Color online) Comparison of predictions at $T=300 \mathrm{~K}$ for (a) the van der Waals and Casimir free energy per unit area where the solid and dashed lines represent the results for pristine graphene obtained by us and in Ref. ${ }^{21}$, respectively (see Ref. ${ }^{21}$ for possible explanations of the discrepancy), and (b) the normalized van der Waals and Casimir pressure where the solid lines represent our results for $\Delta=0.1 \mathrm{eV}, 0.05 \mathrm{eV}$ and $0 \mathrm{eV}$ from bottom to top, respectively, and the dashed lines show the results of Ref $\frac{22}{2}$ for $\Delta=0.1 \mathrm{eV}$ (bottom) and $\Delta=0 \mathrm{eV}$ (top). 